\title{
Acta
Biochimica
Polonica
}

Vol. 46 No. $3 / 1999$

$609-613$

QUARTERLY

\section{Structural polymorphism of telomeres studied by photon correlation spectroscopy ${ }^{\star}$}

\author{
Agnieszka Włodarczyk ${ }^{\mathbb{2}}$, Jacek Gapiński, Adam Patkowski and Andrzej Dobek \\ Faculty of Physics, A. Mickiewicz University, Umultowska 85, 61-614 Poznań, Poland
}

Received: 23 June, 1999

Key words: telomere, photon correlation spectroscopy, chromosome ends

\begin{abstract}
Photon Correlation Spectroseopy (PCS) was used to study the dynamics and structure of Tetrahymena telomeric sequence d(5'-TGGGGT-3') $)_{4}$. Two different modes were observed, corresponding to the following structures: intermolecular (tetramolecular) G-quadruplex and intramolecular (monomeric) G-quartet. Experimental values of translational diffusion coefficients $D_{\mathrm{T}}$ were obtained for each structural form. The value of $D_{\mathrm{T}}$ for the monomer equals to $1.4 \times 10^{6}\left(\mathrm{~cm}^{2} / \mathrm{s}\right)$, while for the tetramolecular structure, to $0.8 \times 10^{6}\left(\mathrm{~cm}^{2} / \mathrm{s}\right)$. The relative weight concentrations of these two forms were analyzed versus the concentration of $\mathrm{NaCl}$ varied from $10 \mathrm{mM}$ to $500 \mathrm{mM}$. The values of experimentally determined diffusion coefficients were compared with those calculated assuming the "bead model" and with the atomic coordinates from the NMR and X-ray crystallographic data. For both structures the experimental and calculated values of $D_{\mathrm{T}}$ were in reasonable agreement. In the entire $\mathrm{NaCl}$ concentration range studied, the contribution of the relative weight concentration of the monomeric telomere form changed from $85 \%$ for $10 \mathrm{mM} \mathrm{NaCl}$ to $60 \%$ for $500 \mathrm{mM}$ NaCl.
\end{abstract}

Telomeres, the specialized DNA structures located at the end of eukaryotic chromosomes, consist of small, tandemly repeated DNA sequences [1-3]. Telomeres vary little in sequence (e.g., TTGGGG in Tetrahymene, TTAGGG in human) but significantly in length
( $<50$ base pairs in Euplotes to $>100$ kilobase pairs in mice). They are known to form specific four-stranded helical conformations: the intermolecular (tetramolecular) G-quadruplex (which we will call a "tetramer") and intramolecular (monomeric) G-quartet

\footnotetext{
"Presented at the symposium "Conformation of peptides, proteins and nucleic acids" held in Rynia, Poland, on 26-29 May, 1999.

${ }^{8}$ Corresponding author: Agnieszka Włodarczyk, tel. (48 61) 827 3265; fax. (48 61) 825 7758; e-mail: wlodar@hoth.amu.edu.pl

Abbreviations: $D_{\mathrm{T}}$, translational diffusion coefficient; d(5'-TGGGGT-3'), repeating telomeric sequence of Tetrahymena; PCS, photon correlation spectroscopy; DLS, dynamic light scattering,
} 
(which we will call a "monomer") stabilized by cyclic hydrogen bonds between four guanines [4-6].

Telomeres play an essential role in maintenance of eukaryotic chromosome within a cell by specifically binding to structural proteins. These proteins cap the ends of linear chromosomes, preventing nucleolytic degradation, end-to-end fusion, irregular recombination, and other events that are normally lethal to the cell. Chromosomal ends progressively shorten with each replication cycle, a process that seems to be linked to the limited proliferative ability of normal somatic cells. The loss of the telomeric tandem eventually leads to the cell death $[5,6]$.

Synthetic DNA oligonucleotides containing a number of copies of the G-rich telomeric sequences have the potential to form intramolecularly folded-back G-quadruplex in solution (Fig. 1a) [7]. Guanine-rich oligonucleotides can also intermolecularly associate and form a four-stranded helical structure in solution, here called the "tetramer", in which each strand has an identical conformation - a parallel, right-handed helix, with all nucleotides in the anti configuration (Fig. 1b) [7]. Twice repeated 3'-terminal G-strand forms a foldedback hairpin that dimerizes to create an antiparallel G-quartet structure in which the nucleotides are alternately syn and anti along each strand [7].

The conformation of G-DNA has been investigated by X-ray crystallography [8], chemical footprinting, strand mixing and cross-linking methods [9], as well as various spectroscopic techniques [10-13]. The formation of fourstranded helical structures has been confirmed for short model oligonucleotides in solution and in the crystalline state $[14,15]$. The G-quartet consists of four guanines in a square planar array stabilized by cyclic hydrogen bondings, each guanine acts as both the donor and acceptor for two hydrogen bonds. The cavity in the G-quartet plays the role of a coordination site for monovalent cations. Each monovalent ion is coordinated by eight guanine $0^{6}$-oxygen atoms from the tetrad planes above and below it. For such divalent cations as: $\mathrm{Sr}^{2+}, \mathrm{Mn}^{2+}, \mathrm{Ca}^{2+}, \mathrm{Zn}^{2+}$, the mechanism of ion binding is different so other structures are assumed ([16], M. Bolten, M. Niermann \& W. Eimer, to be published). Analysis of the number and location of the manganese binding sites in G-DNA in the presence and absence of thrombin, implies that the location of the binding sites of divalent cations is the narrow groove in these quadruplexes [17].

In this paper we have shown, using PCS, that both the single-stranded monomer and four-stranded tetramer telomeric structures coexist in a broad range of $\mathrm{NaCl}$ concentration from 10 to $500 \mathrm{mM}$. The relative concentration of the tetramer increases with increasing salt concentration.

\section{MATERIALS AND METHODS}

The sample of d(5'-TGGGGT- 3 ' $)_{4}$ to be studied was synthesized using the cyanoethylphosphoramide method by The Midland Certified Reagent Company of Midland (Texas, U.S.A.). The sample was purified by means of HPLC and was typically 90-95\% pure. For the DLS measurement, samples were dissolved in $10 \mathrm{mM}$ Tris $/ \mathrm{HCl} \mathrm{pH} 7.3$ buffer, the final sodium concentrations were adjusted by dialysis. The dialyzed samples were filtered through a Millipore $0.22 \mu \mathrm{m}$ pore size filter. The light source was an argon-ion laser operating in the single mode at $\lambda=488 \mathrm{~nm}$ with an output power of $480 \mathrm{~mW}$. The vertically polarized component of scattered light was analyzed by an ALV-5000 digital correlator.

\section{RESULTS AND DISCUSSION}

The values of the translational diffusion coefficients for both single stranded monomer and four-stranded tetramer telomeric structures were calculated assuming the "bead model" [18-19], (E. Sadlik, J. Gapiński, 
a)

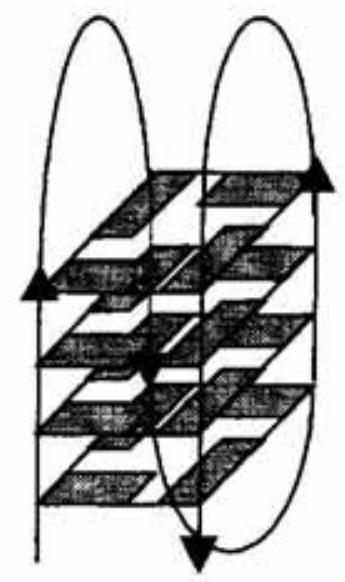

b)

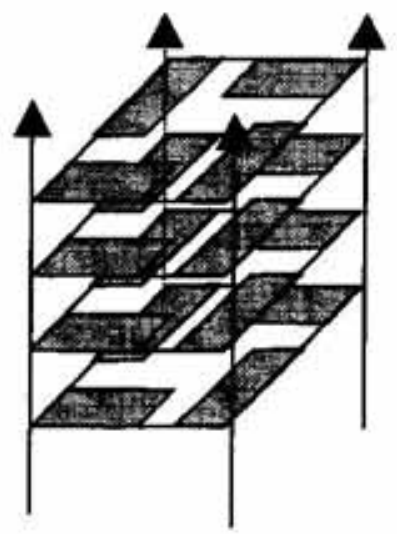

Figure 1. Schematic representation of the G-DNA that can be adopted by telomeric G-strand sequences: a) monomer intramolecular folded-back G-quartet (monomer); b) intermolecular (tetramolecular) G-quadruplex (only one of the four units is shown).

A. Patkowski, A new method of constructing a bead model of biological macromolecules; submitted to Biophys. J.) in which the real structure of a molecule is approximated by a set of beads, whose hydrodynamic properties can be numerically estimated. We used the atomic coordinates of the monomer structure obtained by means of NMR [15] (Brookhaven Protein Data Bank, accession number 186d).
The tetramer telomeric structure was assembled manually by means of the computer program HyperChem from the fragments of identical structure formed by multiple d(5'-TGGGGT-3') units [8] (PDB accession number $352 \mathrm{~d})$. The calculated values of $D_{\mathrm{T}}$ from the hydrodynamic calculations are: for monomer: $D_{\mathrm{T}}=1.46 \times 10^{-6} \mathrm{~cm}^{2} / \mathrm{s}$, for tetramer: $D_{\mathrm{T}}=0.8 \times 10^{-6} \mathrm{~cm}^{2} / \mathrm{s}$. Experimentally measured values of $D$ T are $1.4 \times 10^{6}$ $\left(\mathrm{cm}^{2} / \mathrm{s}\right)$ for the monomer and $0.8 \times 10^{6}\left(\mathrm{~cm}^{2}\right)$ s) for the tetramer.

Photon correlation spectroscopy (PCS) was used to study the dynamics of Tetrahymena telomeric sequence d( $5^{\prime}$-TGGGGT- $\left.3^{\prime}\right)_{4}$ in solution. Two modes were observed in the measured correlation function: the first one was assigned to the folded back single stranded monomer and the second one to the fourstranded tetramer (Fig. $1 \mathrm{a}, \mathrm{b}$ ). The translational diffusion coefficients corresponding to both modes were studied as a function of the telomere and $\mathrm{NaCl}$ concentration. As follows from Fig. 2, the dependence of both diffusion coefficients on the telomere concentration is week. The experimental values of the translational diffusion coefficients for both forms extrapolated to zero telomere concentration are in a reasonable agreement with the calculated values. The diffusion coefficients of both telomeric forms are plotted versus $\mathrm{NaCl}$ concentration in Fig. 3 at a constant telomere concentration of about $3 \mathrm{mg} / \mathrm{ml}$. The salt concentration dependence of these values is also weak. Thus, an unambiguous assignment of each of the two modes of the measured correlation function to one of the telomeric structures is possible at any telomere and salt concentration in the range studied.

The relative contributions (amplitudes) of the components corresponding to the two telomere forms: $A_{\text {mono }}$ and Atetra in the measured correlation functions were estimated from double exponential fits. The amplitude of the process in the correlation function is $A$ $\propto I_{s} \propto c M_{w}$, where $I_{s}$ is the scattered light intensity related to a given process and $M_{w}$ is 


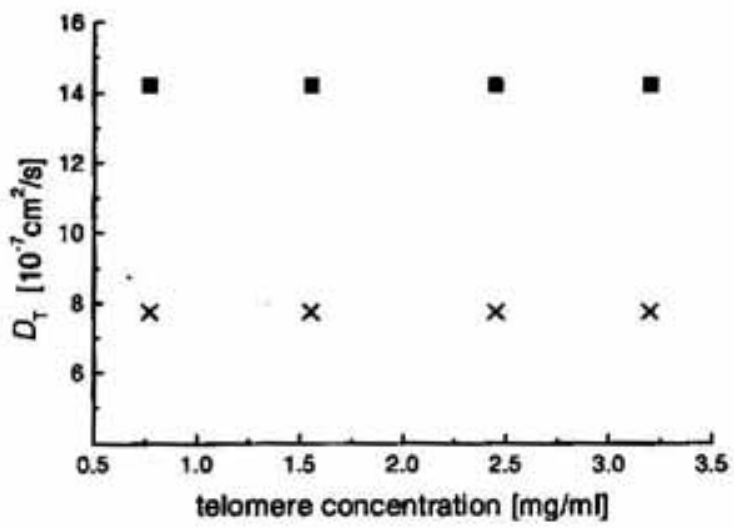

Figure 2. The measured translational diffusion coefficient of the two conformations of the telomere versus the telomere concentration, at $10 \mathrm{mM}$ $\mathrm{NaCl}$.

Symbols: $\mathbf{a}$, monomer; $\times$, tetramer.

the molecular weight of the molecule. Since $M_{w}$ (monomer) $=1 / 4 M_{w}$ (tetramer), the relative weight concentrations (normalized to one) were calculated as $c_{\text {mono }}=A_{\text {mono }} /\left(A_{\text {mono }}\right.$ $\left.+1 / 4 A_{\text {tetra }}\right)$ and $c_{\text {tetra }}=1 / 4 A_{\text {tetra }} /\left(A_{\text {mono }}+1 / 4\right.$ Atetra).

Relative concentrations of both telomeric forms are shown in Fig. 4, which implies that the monomer form of the telomere is the dominant conformation in the entire $\mathrm{NaCl}$ concentration range of $10-500 \mathrm{mM}$. With increasing $\mathrm{NaCl}$ concentration the relative weight concentration of the monomer decreases from $85 \%$ to $60 \%$.

\section{CONCLUSIONS}

Photon correlation spectroscopy was used to identify the d(5'-TGGGGT- $\left.3^{\prime}\right) 4$ telomere conformations present in solution for $\mathrm{NaCl}$ concentrations from 10 to $500 \mathrm{mM}$ and to determine their relative weight concentrations. On the basis of a comparison between the diffusion coefficients measured and calculated assuming the bead model, the two conformational forms were assigned as the singlestranded monomer and the four-stranded tetramer. The single-stranded monomer prevails at all concentrations with relative weight

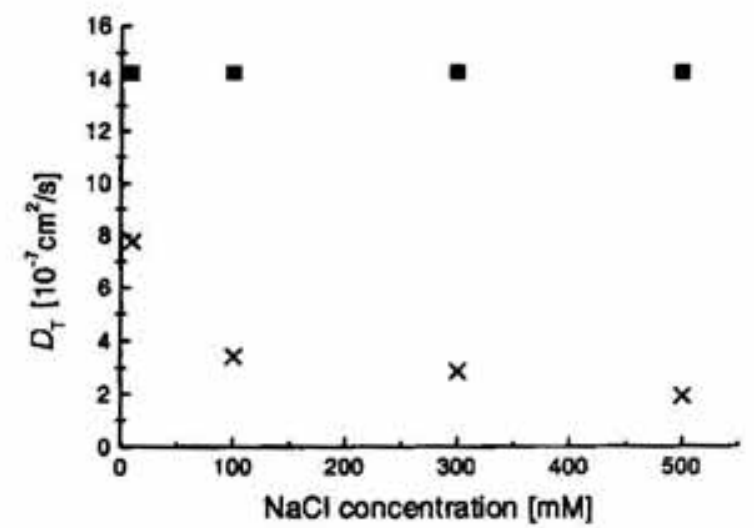

Figure 3. The measured translational diffusion coefficient for the two conformations of the telomere versus $\mathrm{NaCl}$ concentration at a constant telomere concentration of $3 \mathrm{mg} / \mathrm{ml}$.

Symbols: $\mathbf{a}$, monomer; $\times$, tetramer

concentration changing from more than $85 \%$ at low salt to about $60 \%$ at high salt.

In the previous NMR studies [15] performed on $\mathrm{d}_{(}\left(\mathrm{T}_{2} \mathrm{G}_{4}\right)_{4}$ telomere at $100 \mathrm{mM} \mathrm{NaCl}$ and for a much higher telomere concentration it was assumed that the entire sample was in the monomer form. It is not clear to which extent the presence of the tetrameric form of the telomere influences the proposed atomic structure of the monomer. Multiconformational electrophoretic pattern has been previously observed in solutions of a similar telomere: $\mathrm{d}-\left(\mathrm{G}_{4} \mathrm{~T}_{2} \mathrm{G}_{4} \mathrm{~T}_{2} \mathrm{G}_{4} \mathrm{~T}_{2} \mathrm{G}_{4}\right)$ in the presence of $\mathrm{Na}^{+}, \mathrm{K}^{+}$, and $\mathrm{Sr}^{2+}$ ions [16]. This may

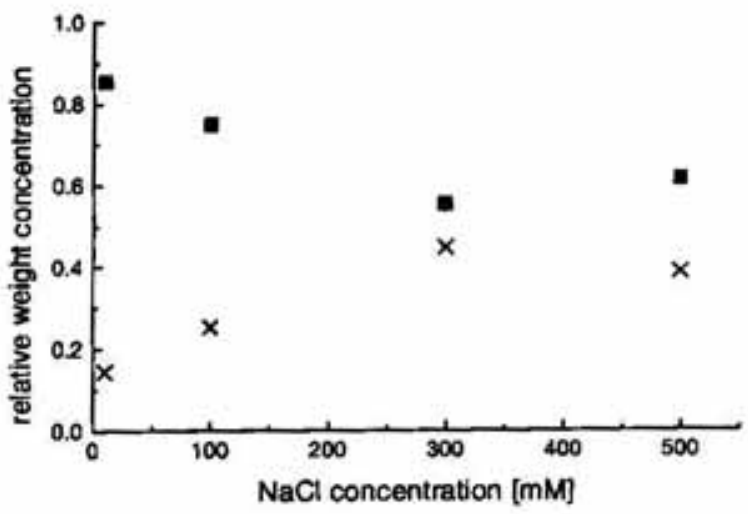

Figure 4. Relative concentrations of the monomer (E) and tetramer $(x)$ telomeric forms.

Telomere concentration is constant and equals about $3 \mathrm{mg} / \mathrm{ml}$. 
indicate that in the tetramer formation from the monomer some intermediate conformational forms are involved. In our study we have not observed these intermediate forms and we cannot say anything about the mechanism of tetramer formation yet.

\section{R E F E R E N C ES}

1. Blackburn, E. \& Szostak, J.W. (1984) The molecular structure of centromeres and telomeres. Annu. Rev. Biochem. 53, 163-194.

2. Rhodes, D. \& Koenig, P. (1997) Recognition of telomeric DNA. Trends Biochem. Sci. 22, 4347.

3. Greider, C.W. \& Blackburn, E. (1985) Identification of a specific telomere terminal transferase activity in Tetrahymena extracts. Cell 43, 405-413.

4. Min, L. \& Qiu, G. (1993) Thermodynamics of G-tetraplex formation by telomeric DNAs. Biochemistry 32, 598-601.

5. Wellinger, R.J. \& Ethier, K. (1996) Evidence for a new step in telomere maintenance. Cell 85, 423-433.

6. Guo-Liang, Y. \& Bardley, J.D. (1990) In vivo alteration of telomere sequences and senescence caused by mutated Tetrahymena telomerase RNAs. Nature 344, 126-131.

7. Rhodes, D. \& Giraldo, R. (1995) Telomere structure and function. Curr. Opin. Struct. Biol. 5, 311-322.

8. Phillips, K. \& Dauter, Z. (1997) The crystal structure of a parallel-stranded guanine tetraplex at $0.95 \AA$ resolution. J. Mol. Biol. 273, 171-182.

9. Sen, D. \& Gilbert, W. (1992) Novel DNA superstructures formed by telomere-like oligomers. Biochemistry 31, 65-70.
10. Aboulela, F. \& Murchie, A.I.H. (1994) Solution structure of a parallel-stranded tetraplex Formed by d(TGGGGT) in the presence of sodium ions by nuclear magnetic resonance spectroscopy. J. Mol Biol. 243, 458-471.

11. Miura, T. \& Thomas, G.J., Jr. (1994) Structural polymorphism of telomere DNA: Inter-quadruplex and duplex-quadruplex conversions probed by Raman spectroscopy. Biochemistry 33, 7848-7856.

12. Wang, Y. \& Patel, D.J. (1993) Solution structure of a parallel-stranded G-quadruplex DNA. J. Mol. Biol. 234, 1171-1183.

13. Wang, Y. \& Patel, D.J. (1992) Guanine residues in $d\left(T_{2} A_{3}\right)$ and $d\left(T_{2} G_{4}\right)$ form parallel -stranded potassium cation stabilized G-quadruplexes with anti glycosidic torsion angles in solution. Biochemistry 31, 8112-8119.

14. Laughlan, G. \& Murchie, A.I.H. (1994) The high-resolution crystal structure of a parallel-stranded guanine tetraplex. Science 265, 520-524.

15. Wang, Y. \& Patel, D.J. (1994) Solution structure of the Tetrahymena telomeric repeat $\mathrm{d}\left(\mathrm{T}_{2} \mathrm{G}_{4}\right)_{4}$ G-tetraplex. Structure 2, 1141-1156.

16. Chen, F.-M. (1992) $\mathrm{Sr}^{2+}$ facilitates intermolecular G-quadruplex formation of telomeric sequences. Biochemistry 31, 3769-3776.

17. Marathias, V.M. \& Wang, K.Y. (1996) Determination of the number and location of the manganese binding sites of DNA quadruplexes in solution by EPR and NMR in the presence and absence of thrombin. J. Mol. Biol. 260, 378-394.

18. Garcia de la Torre, J. \& Navarro, S. (1994) Hydrodynamic properties of a dublehelical model for DNA. Biophys. J. 66, 1573-1579.

19. Garcia de la Torre, J. \& Bloomfield, V.A. (1981) Hydrodynamic properties of complex, rigid, biological macromolecules: Theory and applications. Quart. Rev. Biophys. 14, 81-139. 